\title{
A Reconfigurable Platform for Cognitive Radio
}

\author{
Q. Zhang, G.J.M. Smit, L.T. Smit, A. Kokkeler, F.W. Hoeksema, M. Heskamp \\ University of Twente, Department EEMCS, P.O. Box 217, 7500 AE Enschede, The Netherlands
}

E-mail: Q.Zhang@utwente.nl

\begin{abstract}
Today's rigid spectrum allocation scheme creates a spectrum scarcity problem for future wireless communications. Measurements show that a wide range of the allocated frequency bands are rarely used. Cognitive radio is a novel approach to improve the spectrum usage, which is able to sense the spectrum and adapt its transmission while coexisting with the licensed spectrum user. A reconfigurable radio platform is required to provide enough adaptivity for cognitive radio. In this paper, we propose a cognitive radio system architecture and discuss its possible implementation on a heterogeneous reconfigurable radio platform.
\end{abstract}

Index Terms - Cognitive Radio, Reconfigurable platform, Run time mapping.

\section{INTRODUCTION}

With the growth of future wireless networks, there will be a crisis of spectrum availability under the current spectrum allocation scheme. However, actual measurements show that current spectrum allocation makes the spectrum utilization inefficient because a wide range of the allocated frequency bands are rarely used. This gap between regulatory spectrum allocation and actual usage indicates that a new approach of spectrum usage is needed. Cognitive radio [1], [2] becomes an emerging idea to improve the spectrum utilization. A lower priority secondary user, which exploits cognitive radio, can share the spectrum with the licensed user on a non-interference basis. The conceived cognitive radio system should be able to sense the spectrum to detect the licensed user and adapt its transmission scheme accordingly. It has capabilities including awareness, intelligence, adaptivity and reliability. A cognitive radio system with aforementioned capabilities can be built on a real-time reconfigurable radio platform. This paper will present a prototyped cognitive radio system architecture and discuss its possible implementation on the heterogeneous reconfigurable radio platform.

\section{SYSTEM ARCHITECTURE}

The conceived system architecture focuses on the physical layer of the ISO/OSI protocol stack. We consider that the cognitive radio is not specific to only one set of high layer protocols and thus high layers are not relevant to our discussion. The conceived system architecture consists of two major blocks, namely spectrum scanning and adaptive baseband processing. In the following discussion, we refer the secondary user who applies cognitive radio as the cognitive user opposed to the licensed user who has the legacy right for the spectrum.

\section{A. Spectrum Scanning}

A cognitive radio is able to sense the spectrum in order to identify the available frequency band for transmission. In addition, the cognitive user has a table of frequencies not allowed to use (e.g. reserved for military or astronomy). The spectrum scanning block is aimed to realize this functionality. Although radio resources are available in multiple dimensions such as time, frequency and space, we consider time-frequency as the principle radio resources. When there are activities of a licensed user on certain frequency bands, a relatively high power appears on those bands. We could assume those bands are not available for the cognitive user. A threshold can be set on the power spectrum to detect the licensed user shown in the Figure 1.

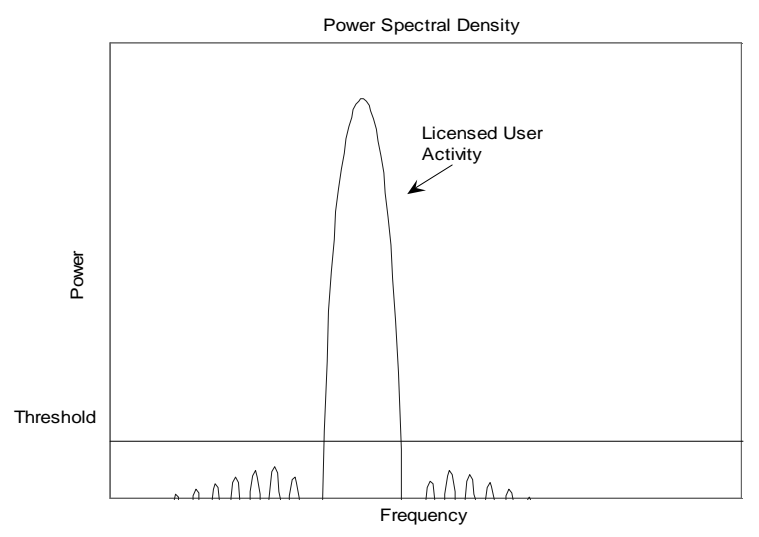

Fig.1. Licensed user detection by power threshold.

In this way, the scanning system is modeled as a spectrum power detector. From implementation point of view it can be approached in a relatively simple way. We propose three different spectrum scanning architectures: scan-based, realtime FFT based and scanning-FFT based.

The scan-based architecture uses only one bandpass filter with a tunable center frequency. During a time interval, it measures the power spectrum in a certain narrow band within the whole spectrum of its interest. In the next time interval, it moves to a different band for a new measurement. This process continues until it has scanned the whole spectrum. This scheme has relatively relaxed requirements for the analog front-end and the $A / D$ converter because the targeted bandwidth is relatively 
small. However, it may take a long time to go through the whole spectrum.

The real-time FFT based architecture digitizes the whole spectrum so that an FFT algorithm can be efficiently used to obtain the information in the frequency domain. However, it requires an analog front-end which is able to operate on a wide frequency band. In addition, a high speed and lower power A/D converter is also needed.

The scanning-FFT based architecture is actually the combination of the two methods. It uses a real-time FFT based method on a fraction of the whole spectrum span. It performs measurements on different fractions until the whole spectrum is covered.

The spectrum scanning is done by each cognitive user in a distributed fashion, where the local spectrum usage information is obtained. Then it is necessary to exchange and combine the local information to generate the global spectrum usage pattern in the communication group of cognitive users.

\section{B. Adaptive Baseband Processing}

When the spectrum usage pattern is generated, the cognitive user should adapt its transmission scheme accordingly while avoiding interference to the licensed user. Our conceived adaptive baseband processing system is an OFDM like system across the entire band of interest. It could avoid the licensed user by adaptively loading the information on different carriers, see Figure 2. During a certain time period, the information can be loaded to the carrier bands not occupied by the licensed user. When the spectrum usage changes over time, the information loading pattern will also change accordingly.

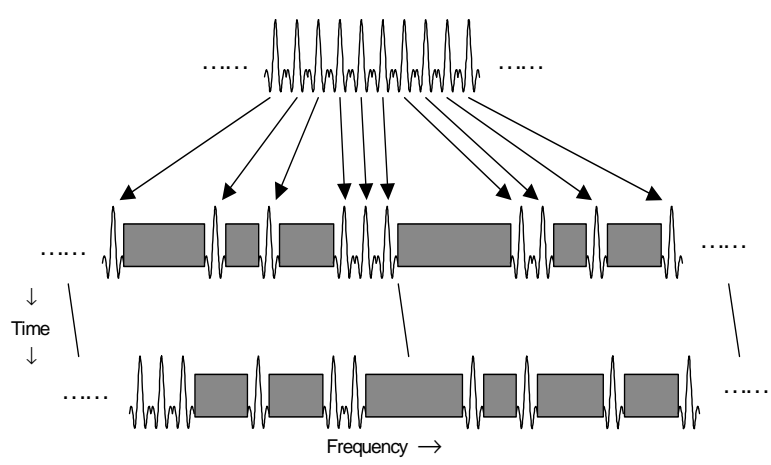

Fig.2. Adaptive baseband system

However, the traditional OFDM system is not optimal for cognitive radio because there is a power leakage to adjacent carriers which can cause potential interference to the licensed user. In [3], two countermeasures to address this problem have been proposed: proper windowing and adaptive deactivation of adjacent carriers. Therefore, the OFDM system for cognitive radio is an open issue for further investigation. The channel estimation is also an important functionality for the baseband processing system because the channel conditions often change. Although the detailed architecture of the adaptive baseband processing part is not clear, we foresee it should have enough adaptivity in order to optimally use the available spectrum. To give an indication of the adaptivity of the system, the following major adaptive parameters are given:

- The number of carriers. Given the frequency range, this parameter is mainly determined by the allowed inter-carrier interference (ICI).

- The prefix length of OFDM symbols. This parameter is mainly determined by the delay spread of the channel which causes inter-symbol interference (ISI).

- Bit loading per carrier. For each carrier, the bitloading is mainly determined by the SNR of this carrier band in which the licensed user is not active. When detecting a licensed user on a carrier band, cognitive radio loads that carrier with zero bits, which is equal to switching off the carrier.

In addition, cognitive radio should be able to operate at different channel codes and power levels. It is also possible to incorporate a MIMO scheme.

\section{RECONFIGURABLE RADIO PLATFORM}

A reconfigurable radio is able to change its application, parameters and structure while the radio is in operation. As mentioned before, the cognitive radio has to adapt to the wireless channel condition. Thus the reconfigurable radio platform can be used to realize the adaptivity required by the cognitive radio. Our proposed reconfigurable radio platform is a heterogeneous system-on-chip (SoC) as shown in Figure 3.

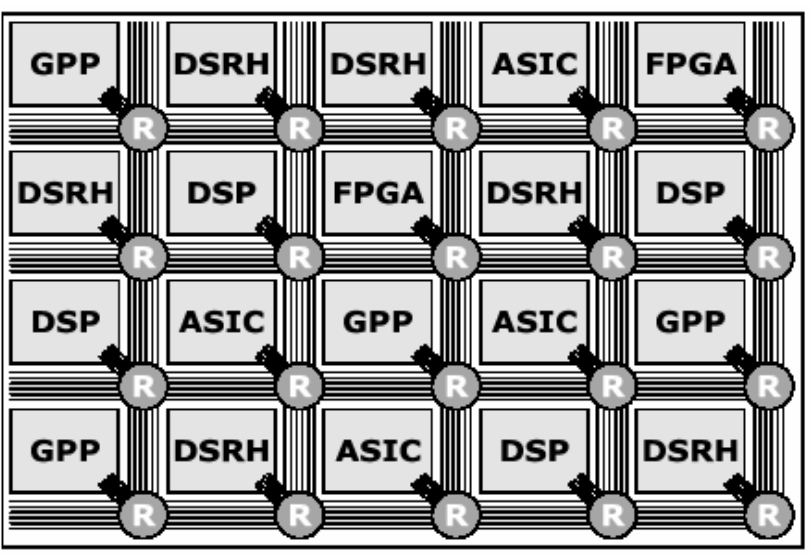

Fig.3. Heterogeneous system-on-chip 
It consists of different types of processing tiles including general purpose processor (GPP), domain specific reconfigurable hardware (DSRH), DSP, FPGA and ASIC. DSRH is a relatively new term, which means the processing element can be reconfigured within the specific algorithm domain (e.g. FFT and FIR filtering). The MONTIUM [4] developed in our group is an example of the DSRH. The MONTIUM targets the digital signal processing (DSP) algorithm domain which is the building element for wireless communications. In the previous work [5] [6] [7], various communication algorithms including FFT, FIR and Viterbi decoding have been mapped onto the MONTIUM. The implementation results on the MONTIUM shows its adaptivity to different algorithms and system parameters with more energy efficiency than GPP and FPGA. We think the DSRH like MONTIUM is a promising solution for future energy-efficient adaptive wireless communications. These aforementioned processing tiles are connected by the router-based network-on-chip (NoC) which is also reconfigurable. This heterogeneous reconfigurable system has several advantages over a single processor reconfigurable platform. First, it can provide reconfigurability in a more energy-efficient manner, which is a desirable feature for mobile devices. An application can be performed on the most appropriate processing tile based on the nature of the application. For example, computationally intensive applications like FFTs, can be performed on an ASIC or DSRH while control intensive applications can be performed on a GPP. Second, dynamic partial reconfiguration can be done on the local tile so that there is no need to load the entire new configuration. Thus, the configuration time is reduced to meet the timing constraint. Third, unused tiles can be switched off to save energy.

However, the design methodology of the heterogeneous system-on-chip is different from uniprocessors or homogeneous systems. The designer has to partition the application into a process graph with communicating functional processes. In the following section, we will introduce a new design methodology.

\section{IMPLEMENTING COGNITIVE RADIO ON A RECONFIGURABLE PLATFORM}

In this section, we propose a new design methodology for cognitive radio featuring mapping applications to processors at run time. The design flow diagram is shown in Figure 4.

The design flow starts with the high level system description in software. The application algorithms can be written as a collection of functional processes which communicate via channels. Those functional processes can be modeled as the process graph. An example in Figure 5 shows the process graph for the baseband OFDM receiver. Besides the description of the application, Quality of
Service (QoS) requirements in the application such as throughput, latency, maximum energy consumption have to be specified.

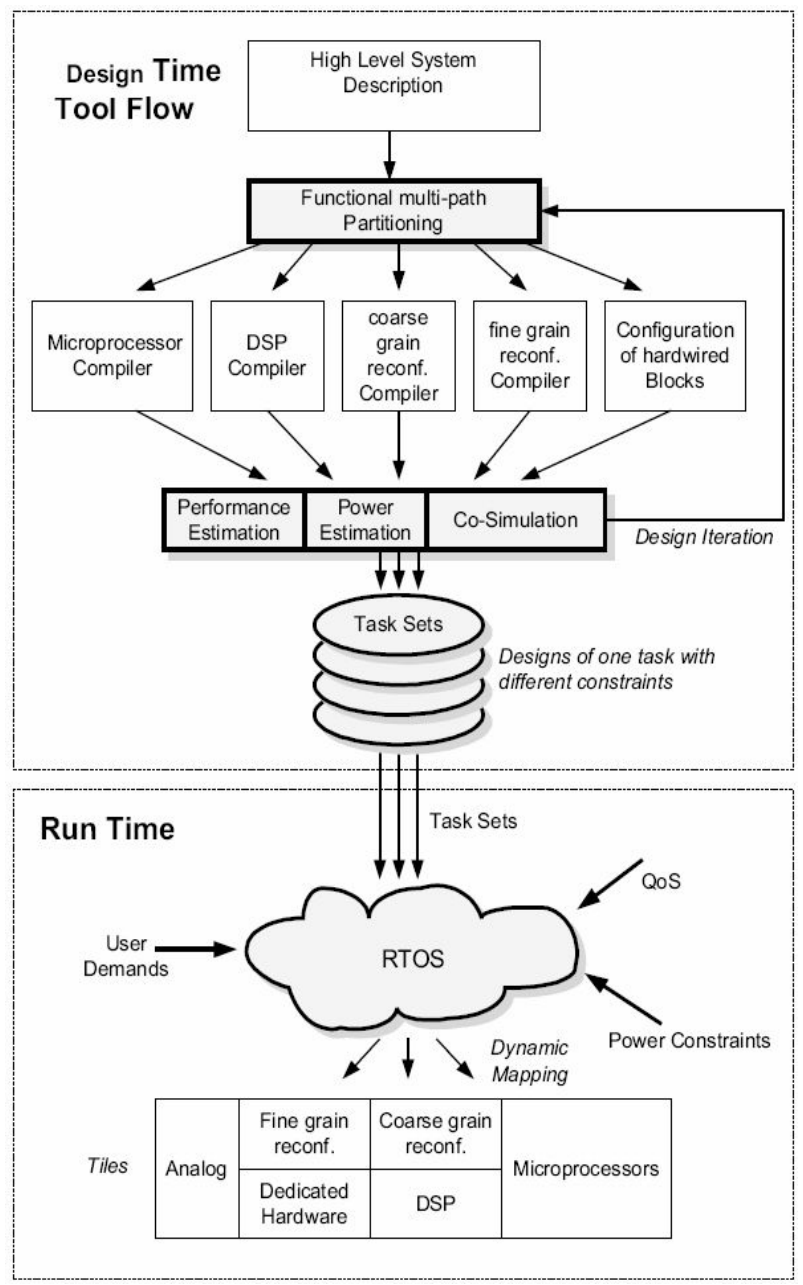

Fig.4. Design flow of cognitive radio

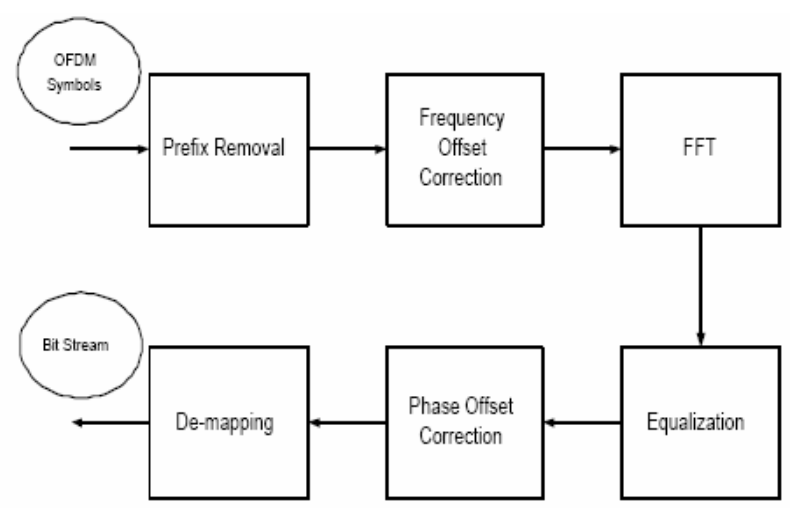

Fig.5. Process graph for baseband OFDM receiver 
Each individual functional process is compiled or synthesized to one or more processing tiles. The processes implementations will be annotated with performance characteristics (e.g. number of clock cycles, energy consumption, memory requirements, and average load on a processing element). A modified functional process partitioning may be generated in order to achieve optimal performance characteristics. These performance characteristics form a library to be used to generate a mapping solution.

On the other hand, the architecture description of the heterogeneous SoC has to be done. For each tile a number of characteristics are provided such as the type of the tile, the amount of available memory, the clock frequency, etc. The characteristics of the NoC are also provided, such as the topology of the network, the frequency of the clock of the network, latency per router, etc.

Based on the information from the library of the performance characteristics, the traditional design-time methods find an optimal mapping to map the processes to processing tiles. However, this method may not be effective for cognitive radio which could change or add constraints in real time according to the varying wireless channel conditions. This implies a new method of mapping should be used for cognitive radio. We propose that process mapping can be done at run-time by a real time operating system (RTOS). This RTOS is responsible for dynamically selecting an optimal mapping based on the performance characteristics to map processes to the processing tiles while satisfying several constraints including QoS, timing constraint, and energy constraint. It also takes the processors' capacity and NoC capacity into consideration. When the change of the wireless channel condition occurs, it will generate new system constraints which trigger a new mapping done by the RTOS.

The run-time mapping algorithm finds an optimal solution in the search space under certain constraints. In our case, the major optimization objective is to minimize the energy consumption of the $\mathrm{SoC}$, while still providing the required QoS. Research in the area of scheduling and optimization theory has many similarities with the problem we are considering. There are heuristic techniques for finding optimal solutions such as greedy algorithms, simulated annealing and genetic algorithms. However, we realize these algorithms may be too computationally expensive to find an optimal mapping because the RTOS has to search all possibilities at run-time. Instead, a minimum weight algorithm in [8] is proposed to find a near-optimal mapping. Some preliminary results in [8] demonstrate its effectiveness to give a near optimal solution in a reasonable short computational time. This algorithm shows its potential application on the RTOS of cognitive radio.

\section{CONCLUSION}

In this paper we proposed a system concept of cognitive radio and discussed its possible implementation on a heterogeneous reconfigurable radio platform. Cognitive radio is aimed to solve the spectrum scarcity problem caused by the regulatory spectrum allocation. It may be a good candidate for future mobile systems considering that more and more wireless systems will compete with each other to use the spectrum. However, cognitive radio is only in its initial stage and many questions need to be answered. Many new research projects start to investigate this topic. Our research is under the Adaptive Ad-hoc Freeband (AAF) project [9] sponsored by Freeband Organization in the Netherlands. In future, we aim to come up with a detailed system architecture and sets of algorithms for cognitive radio. The ultimate goal is to build a heterogeneous reconfigurable radio platform to demonstrate the cognitive radio functionalities.

\section{ACKNOWLEDGEMENT}

This research is conducted within Adaptive Ad-hoc Freeband (AAF) project supported by Freeband Organization, the Netherlands.

\section{REFERENCES}

[1] Joseph Mitola III. "Cognitive Radio an Integrated Agent Architecture for Software Defined Radio", PhD Thesis, KHT Royal Institute of Technology, Sweden, 2000

[2] Simon Haykin, "Cognitive Radio: Brain-Empowered Wireless Communications", IEEE Transaction on Selected Areas in Communications, Feb. 2005

[3] Timo Weiss, Joerg Hillenbrand, Albert Krohn, Friedrich K. Jondral, "Mutual Interference in OFDM-based Spectrum Pooling Systems", Proc. of the Vehicular Technology Conference, Italy, 2004

[4] Paul Heysters, "Coarse Grained Reconfigurable Processors-Flexibility meets Efficiency", PhD Thesis, University of Twente, The Netherlands, 2004

[5] Gerard Rauwerda, G.J.M. Smit, L.F.W. van Hoesel, P.M. Heysters, "Mapping Wireless Communication Algorithms to a Reconfigurable Architecture", Proceedings of the International Conference on Engineering of Reconfigurable Systems and Algorithms, USA, 2003

[6] Gerard Rauwerda, Gerard J.M. Smit, Werner Brugger, "Implementing an Adaptive Viterbi Algorithm in Coarse-Grained Reconfigurable Hardware", Proceedings of the International Conference on Engineering of Reconfigurable Systems and Algorithms, USA, 2005

[7] Arnaud Rivaton, Jérôme Quevremont, Qiwei Zhang, Pascal. Wolkotte, Gerard. Smit, "Implementing Non Power-of-Two FFTs on Coarse-Grain Reconfigurable 
Architectures" Accepted for the International Symposium on System-on-Chip (SoC2005), Tampere, Finland, November 15-17, 2005

[8] Lodewijk Smit, Gerard J. M. Smit and Johann L. Hurink, Hajo Broersma, Daniel Paulusma, Pascal T. Wolkotte, "Run-time mapping of applications to a heterogeneous reconfigurable tiled system on chip architecture", Proc. of the International Conference on Field-Programmable Technology, 2004

[9] Freeband website: www.freeband.nl 